\title{
RESPONSABILIDAD SOCIAL CORPORATIVA: ESTRATEGIA EMPRESARIAL PARA EL DESARROLLO SOSTENIBLE
}

\section{CORPORATE SOCIAL RESPONSIBILITY: BUSINESS STRATEGY FOR SUSTAINABLE DEVELOPMENT}

\author{
Vásquez-Giler Maritza ${ }^{1 *}$; Zambrano-Vera Teddy²; Muñoz-Menéndez María Belén ${ }^{3}$ \\ ${ }^{1}$ Directora de Postgrado de la Universidad Laica Eloy Alfaro de Manabí, ULEAM. \\ Manta, Ecuador \\ ${ }^{2}$ Fiscal de la Universidad Laica Eloy Alfaro de Manabí, ULEAM. Manta, Ecuador \\ ${ }^{3}$ Directora de Educación Continua de la Universidad Laica Eloy Alfaro de Manabí, \\ Manta, Ecuador
}

${ }^{*}$ Correo: post-mari20@gmail.com

\begin{abstract}
Resumen
La Responsabilidad Social Corporativa es actualmente una temática de constante discusión y análisis, puesto que desde distintas perspectivas se busca posicionar este concepto no solo como un mecanismo empresarial de ser agradables ante la sociedad o la comunidad; sino más bien como una estrategia de crecimiento empresarial a mediano y largo plazo. El presente trabajo tiene como objetivo analizar los conceptos, origen y estado actual de las empresas socialmente responsables, para de esta forma establecer pautas que conviertan a las organizaciones en comunidad. Se analizan los criterios que dan soporte a una estructura empresarial responsable con la colectividad. Además, se analiza el grado de responsabilidad con el que se manejan las empresas latinoamericanas en el contexto mundial. Se concluye que si bien las empresas son conscientes de la importancia de la RSC y su consecuente impacto positivo aún quedan tareas pendientes para posicionar políticas de este tipo entre los empresarios y ejecutivos. Las instituciones deben ser concebidas como comunidad, partiendo de valores morales y éticos que fomentan el desarrollo de una identidad replanteada a partir de la apertura hacia las necesidades de los demás.
\end{abstract}

Palabras clave: comunidad, ambiente, organización, criterios, responsabilidad.

\begin{abstract}
Corporate Social Responsibility is currently a subject of constant discussion and analysis, since from different perspectives it is sought to position this concept not only as a business mechanism of being pleasant to society or the community; but rather as a medium and long term business growth strategy. This paper aims to analyze the concepts, origin and current status of socially responsible companies, in order to establish guidelines that convert organizations into a community. The criteria that support a responsible business structure with the community are analyzed. In addition, the degree of responsibility with which Latin American companies are managed in the global context is analyzed. It is concluded that although companies are aware of the importance of CSR and its consequent positive impact, there are still pending tasks to position policies of this type among businessmen and executives. Institutions must be conceived as a community, based on moral and ethical values that foster the development of a rethinking identity based on openness towards the needs of others.
\end{abstract}

Keywords: community, environment, organization, criteria, responsibility.

Información del manuscrito:

Fecha de recepción: 28 de agosto de 2019

Fecha de aceptación: 16 de diciembre de 2019

Fecha de publicación: 10 de enero de 2020 


\section{Introducción}

La responsabilidad social corporativa (RSC) es un campo del conocimiento que se explora y actualiza continuamente, puesto que se buscan puntos de mejora para potenciar esta actividad en la planificación de las actividades estratégicas de empresas e industrias. Varios autores proponen las ventajas de la gestión de empresas basada en principios de responsabilidad social; así como aportaciones teóricas que implican que las organizaciones promuevan una misión y visión integral que asocien la rentabilidad con beneficios y mejoras para la comunidad en aspectos sociales, ambientales y culturales. Las áreas de acción más significativas en la RSC se basan en la vinculación con la sociedad en términos educativos, deporte, cultura y cuidado al medio ambiente. Evidentemente, el foco de atención y los primeros beneficiados son los empleados y trabajadores de la organización (Chirinos, Fernández \& Sánchez, 2012).

La sociedad experimenta cambios radicales que requieren un mayor compromiso del ser humano con su entorno. Desde la arista social, en muchas empresas se presentan condiciones desfavorables para los trabajadores en términos discriminatorios; además, innumerables actos de corrupción en empresas públicas y privadas que perjudican a millones de personas en todo el mundo, puesto que, a pesar de la permanente actualización de las leyes y directrices para mitigar esta problemática, se siguen presentando casos en todos los rincones del mundo (Lindskog, Brege \& Brehmer, 2010). Asimismo, en la esfera medioambiental, las industrias manufactureras han generado severas consecuencias sobre los ecosistemas; por citar el caso del derrame de hidrocarburos en el Golfo de México el 2010, en el que la compañía British Petroleum causó un desastre medioambiental, cuyos efectos se cuantifican en los $1550 \mathrm{~km}^{2}$ en las costas de Alabama, Luisiana y Mississippi (Avendaño, 2013). En otro contexto, Ecuador es un país exportador de petróleo que ha sufrido los efectos de la extracción de este recurso, puesto que Buccina, Chene \& Gramlich (2013) contabilizaron los impactos ambientales de las operaciones petroleras y determinaron que gran parte de las plantas de extracción 
hidrocarburífera desarrollan sus actividades a través de prácticas que han contaminado los recursos naturales de la amazonia ecuatoriana.

Por otra parte, cabe mencionar que la responsabilidad social es en la actualidad una necesidad convertida en estrategia de las empresas que buscan adaptar ventajas competitivas en modo comunicativo y de respuesta a las necesidades organizacionales aplicadas al desarrollo empresarial y social (Chirinos et al., 2012).

El presente manuscrito tiene como objetivo discutir las estrategias de responsabilidad social en la empresa moderna que busca garantizar un crecimiento sostenible y sustentable en aspectos sociales, económicos y ambientales.

\section{Origen de la Responsabilidad Social Corporativa}

El concepto de empresa nació aislado de las ciencias sociales; no obstante, a partir de 1990, esta área del conocimiento incluyó a la empresa y el comercio como subáreas relevantes (Toro, 2006). De modo que en los últimos años 30 años se han generado varios debates y constantes discusiones en base a la función social que cumplen las empresas con la comunidad. Actualmente, las organizaciones han incorporado a su gestión los avances en materia de Responsabilidad Social Corporativa, sin embargo, es notorio el déficit en cuanto a conceptos y estrategias sólidas y consensuadas entre varios autores (Kirschner, 2005).

\section{RSC en el contexto latino}

En Latinoamérica existen diferencias entre la percepción de usuarios/consumidores y administradores de empresas e industrias acerca de la influencia e importancia de la responsabilidad social. Por una parte, se propone que los consumidores están dispuestos a asumir un valor adicional por un producto o servicio que fue generado mediante la aplicación de mecanismos social y ambientalmente responsables, mientras tanto, desde la arista de los ejecutivos, se concluye que un $66 \%$ de los administradores de empresas señalan que esta opción es poco factible o rentable y que por consiguiente, los potenciales compradores no aceptarían productos con un valor adicional 
debido a la implantación de la gestión de RSC (Kowszyk, Covarrubias \& García, 2011).

En este sentido, KLP es el Fondo de Previsión Noruego, que mantiene estándares y criterios éticos que rigen sus inversiones; además, publica oficialmente una lista de las empresas excluidas basada en parámetros de acuerdos internacionales en pro de los derechos humanos, corrupción y medio ambiente (Brammer \& Millington, 2003). La mayoría de empresas $(61 \%)$ excluidas por KLP han incurrido en violaciones a los acuerdos mencionados, en territorio latinoamericano. Las empresas de extracción de petróleo encabezan esta lista.

\section{RSC: oportunidad de crecimiento empresarial}

Actualmente, el concepto de responsabilidad social corporativa busca introducirse no solo como un mecanismo para gestionar la actividad e impacto social $y$ ambiental de una empresa, sino también como un medio para potenciar la actividad comercial de la organización, en lo que se denomina comercio ético. Klausen (2000) propone que las empresas que adoptan políticas de RSC son más rentables que las que omiten estas medidas, asociando este comportamiento con una administración empresarial moderna, empática y con un alto sentido de pertenencia con el medio.

Por otra parte, el fondo ético más analizado: Dow Jones Sustainability Index (DJSI) ha generado mayores pérdidas que el Dow Jones General Index, el cual no tiene un enfoque prioritario hacia la responsabilidad social. Es notorio que existe diversidad de evidencias y aún no se establece un consenso respecto a las ventajas comerciales de las empresas socialmente responsables; sin embargo, muchos estudios empiezan a demostrar y a validar el crecimiento sostenible que alcanzan las organizaciones manejadas bajo principios éticos y responsables (Holme \& Watts, 2002).

\subsection{Criterios de las empresas socialmente responsables}

A continuación, se plantean los criterios propuestos por la organización CEMEFI. Según Porto \& Castromán (2006) se deben cumplir al menos los siguientes criterios para garantizar una 
administración

empresarial

responsable con el medio ambiente y la sociedad:

\section{a) Fomentar una óptima calidad de} vida dentro de la organización. La empresa debe asegurar la seguridad y salud de los trabajadores, por lo tanto, debe brindar condiciones favorables para el trabajo, así como un buen clima laboral y organizacional. En este sentido, la organización debe capacitar permanentemente a los trabajadores, evitar el sobrecargo de trabajo, pagar honorarios profesionales en función de la formación y experiencia, identificar y evaluar riesgos laborales, inspeccionar y rediseñar puestos de trabajo; puesto que estas son estrategias que provocan un efecto positivo en el desarrollo de la institución.

b) Código de ética. La administración de una empresa socialmente responsable mantiene relaciones fraternas con clientes y proveedores, lo cual proyecta una mejor imagen y mayor confianza de potenciales clientes; por lo tanto, debe constituirse en una relación ganar-ganar (Barroso, 2008). c) Vinculación con la comunidad.

El crecimiento de la organización debe sustentarse en acciones que generen desarrollo en la comunidad, con aportes significativos en materia de bienestar social. Es por testa razón que desde la alta dirección debe gestionarse la creación de políticas, misión y visión que fomenten la participación activa en programas como donaciones, becas para niños y adolescentes, inclusión de adultos mayores en actividades lúdicas y obras de beneficencia.

d) Cuidado y saneamiento ambiental. Una organización comprometida con la responsabilidad social, tiene un manejo administrativo que toma acciones que permitan preservar el medio que los rodea. Además, siendo el caso de que la industria genere residuos, debe gestionar su tratamiento y descarga sin alterar las condiciones actuales del entorno en base a las normativas ambientales de carácter legal que rigen en cada país.

En Ecuador el Ministerio del Ambiente (MAE) propone los requisitos ambientales que deben seguirse tanto a nivel empresarial como doméstico. Entre otras 
funciones, el MAE controla las descargas de residuos líquidos y determinó que por año se superan los 70 procesos legales por descargas de residuos en las fuentes naturales de agua (2017).

\section{Conclusiones}

Los criterios (4) planteados por CEMEFI deben ser cumplidos sin excepción por las empresas que buscan implantar una gestión de manejo responsable con la sociedad $y$ el medio ambiente. Estos lineamientos son estándares y no deben ser sesgados en función de las necesidades o disponibilidad de la empresa.

Se concluye que si bien las empresas son conscientes de la importancia de la RSC y su consecuente impacto positivo aún queda mucho por hacer para posicionar políticas de este tipo entre los empresarios y ejecutivos que apuntan tan solo al crecimiento económico de su organización. Las organizaciones deben ser concebidas como comunidad, partiendo de valores morales y éticos que fomentan el desarrollo de una identidad replanteada a partir de la apertura a las necesidades de los demás.
Finalmente, cabe indicar que las empresas deben realizar un manejo técnico y sustentable de los recursos, además, fomentar en sus trabajadores, proveedores y clientes al cuidado y preservación del ambiente de forma constante y voluntaria.

\section{Bibliografía}

Avendaño, W. R. (2013). Responsabilidad social (RS) y responsabilidad social corporativa (RSC): una nueva perspectiva para las empresas. Revista lasallista de investigación, 10(1), 15.

Barroso, F. (2008). Ganancia máxima o ganancia óptima. Desarrollo Empresarial. Año XI, núm. 110, 35-38.

Brammer, S., \& Millington, A. (2003). The effect of stakeholder preferences, organizational structure and industry type on corporate community involvement. Journal of Business ethics, 45(3), 213226.

Buccina, S., Chene, D., \& Gramlich, J. (2013, June). Accounting for the environmental impacts of Texaco's operations in Ecuador: Chevron's contingent environmental liability disclosures. In Accounting Forum, 37, 2, 110123). 
https://doi.org/10.1016/j.accfo r.2013.04.003

Chirinos, M. E., Fernández, L., \& Sánchez, G. (2012). Responsabilidad empresarial o empresas socialmente responsables. Razón y palabra, 17(81).

Holme, R. \& Watts, P. (2002). Corporate Social Responsibility: Making Good Business Sense. Geneva, Switzerland: World Business Report for Sustainable Development.

Kirschner, A. (2005). La Responsabilidad Social de la empresa. En: Nueva sociedad. p. 202.

Klausen, T. (2000). Etiske Investeringer. Rappport 16:2000, Oslo: Institutt for Samfunnsforskning.

Kowszyk, Y., Covarrubias, A. \& García, L. (2011). El estado de la responsabilidad social empresarial en América Latina 2011. Percepciones de consumidores y ejecutivos de empresas. Forum Empresa. Disponible en: http://www.bibliotecavirtualrs. com/wpcontent/uploads/2011/12/esta do-rse-2011-libro.pdf

Lindskog, H., Brege, S., \& Brehmer, P. O. (2010). Corruption in public procurement and private sector purchasing. Journal of Organisational Transformation \& Social Change, 7(2), 167-188. https://doi.org/10.1386/jots.7. 2.167_1

MAE. (2017). Las descargas de aguas residuales son controladas por el Ministerio del Ambiente. Ministerio del Ambiente, Ecuador. Disponible en: http://www.ambiente.gob.ec/l as-descargas-deaguasresidualessoncontroladas-por-elministeriodel-ambiente/. Revisado: 4 de diciembre de 2019.

Porto, N. \& J. Castromán (2006). Responsabilidad social: un análisis de la situación actual en México y España. Contaduría y Administración, 220, 67-87.

Toro, D. (2006). El enfoque estratégico de la responsabilidad social corporativa: revisión de la literatura académica. El enfoque estratégico de la responsabilidad social corporativa: revisión de la literatura académica. Intangible Capital, 2, 14, 338358. 